\title{
A Randomized Controlled Trial to Examine the Feasibility and Preliminary Efficacy of a Digital Mindfulness-Based Therapy for Improving Insomnia Symptoms
}

\author{
Lucinda Kennett ${ }^{1} \cdot$ Bei Bei $^{1} \cdot$ Melinda L. Jackson ${ }^{1}$ \\ Accepted: 21 July 2021 / Published online: 6 August 2021 \\ (c) The Author(s), under exclusive licence to Springer Science+Business Media, LLC, part of Springer Nature 2021
}

\begin{abstract}
Objectives Insomnia has widespread negative implications for health and well-being. Online delivery of mindfulness-based therapy for insomnia (MBTI) has not previously been evaluated. This study investigated the feasibility and preliminary efficacy of a digital MBTI program for improving insomnia symptoms. It was hypothesized that a 6-week digital MBTI program would reduce insomnia symptoms and pre-sleep arousal and improve mood, compared to a waitlist control condition. Methods Twenty-seven participants $(\mathrm{M}=29.44, \mathrm{SD}=11.97$ years) experiencing insomnia symptoms (insomnia severity index [ISI] $\geq 8$ ) were randomized to either a 6-week intervention or waitlist condition. Participants completed the ISI, presleep arousal scale (PSAS), and the positive and negative affect schedule at baseline, mid-, and post-study. Feasibility was assessed across four domains (acceptability, implementation, practicality, and preliminary efficacy) using self-reports, attrition, program completions, and module completions.

Results Feasibility data for the intervention indicated that there was $22 \%$ attrition, and $79 \%$ of the modules were completed. There were significantly greater reductions in the severity of insomnia symptoms $(p<.001)$ and both cognitive $(p=.03)$ and somatic $(p=.02)$ subscales of the PSAS, at post-intervention compared to the waitlist group. There were no significant group differences in mood.

Conclusions This study provides preliminary evidence of the feasibility and efficacy of a digital MBTI, which may assist in the broader dissemination of insomnia treatment.

Trial Registration Australian and New Zealand Clinical Trials Registry: ACTRN12620000398909
\end{abstract}

Keywords Sleep · Cognitive behavioral therapy for insomnia $\cdot$ Digital mindfulness-based therapy $\cdot$ Pre-sleep arousal ·

Mood

Insomnia is a highly prevalent sleep disorder, with between 10 and $20 \%$ of adults experiencing insomnia disorder at any point in time (Chung et al. 2015) and 30 and 45\% experiencing sub-clinical symptoms (Adams et al. 2017). Insomnia is characterized by sleep disturbances at least three times a week, including difficulty falling or staying asleep and waking too early, despite adequate opportunity to sleep, and is accompanied by significant daytime impairment or distress (American Psychiatric Association 2013). When left untreated, insomnia can have widespread negative mental

Melinda L. Jackson

melinda.jackson@monash.edu

School of Psychological Sciences, Turner Institute for Brain and Mental Health, Monash University, 18 Innovation Walk, Clayton, Melbourne, VIC 3001, Australia and physical health implications, including a significant risk factor for development of worsening of psychiatric disorders (Walsh 2004).

While there are multiple theories for the etiology of insomnia, there is a consensus that heightened arousal interferes with sleep. Morin et al.'s (1993) cognitive behavioral model proposes that individuals with insomnia have heightened levels of physiological, cognitive, and emotional arousal that creates a cycle that maintains insomnia. Ong et al. (2012) extended models of arousal to include primary and secondary arousal. Primary arousal refers to cognitive activity that directly interferes with sleep, while secondary arousal refers to the attention and emotional biases towards negative sleep-related cognitions. Ong et al.'s (2012) metacognitive model of insomnia proposes that it is the interaction of these two levels that maintains insomnia. 
Despite the considerable impact of insomnia on health and well-being, there remains a lack of access to treatment (Ree et al., 2017). Cognitive behavioral therapy for insomnia (CBTi) is considered the first-line treatment for insomnia (Ree et al., 2017). Numerous studies have demonstrated CBTi as an effective, non-invasive treatment for reducing insomnia symptoms (Trauer et al., 2015). The efficacy of digital CBTi programs has also been demonstrated across a number of randomized trials (Luik et al., 2017; Seyffert et al., 2016). Although 30-40\% of individuals achieve full remission after in-person CBTi (Morin et al., 2009), CBTi may not be effective for everyone (Garland et al., 2016). Interestingly, individuals who continue to experience insomnia symptoms after CBTi also often experience residual cognitive arousal (e.g., rumination, worry), which may explain the low remission rates (Kalmbach et al. 2019). An adjunct therapy, which may address this limitation of CBTi, incorporates mindfulness.

Mindfulness refers to a state of conscious, intentional, and non-judgmental awareness of the present moment experiences (Gong et al. 2016). Training individuals in mindfulness involves cultivating an intentional awareness to internal and external present moment experiences, often through meditation practices (Baer et al., 2004). Mindfulness-based therapy for Insomnia (MBTI), which has received growing interest in recent years, integrates mindfulness techniques with the behavioral components of CBTi (Ong et al., 2012). Combining mindfulness with elements of CBTi may have additional benefits as they are theorized to work through different mechanisms (Ong et al., 2012). While the cognitive component of CBTi challenges and changes dysfunctional thought content (Ong et al., 2014), MBTI alters the relationships with these thoughts through mindfulness (Ong \& Smith, 2017). In addition, an increased awareness and nonjudgmental stance in individuals with insomnia is theorized to help individuals shift from reactive to adaptive responses towards their insomnia symptoms, to reduce sleep disturbances, and to effectively manage emotional reactions (Ong et al., 2012).

There is growing evidence that MBTI is an effective treatment for reducing the severity of insomnia symptoms in individuals with insomnia (Ong et al., 2008, 2009, 2014). First in-treatment development studies, a 6-week group-based MBTI was found to significantly reduce insomnia severity index (ISI) scores in individuals with insomnia (Ong et al., 2008, 2009). The long-term benefits of an 8-week MBTI, delivered in a group format, were subsequently confirmed in a controlled trial (Ong et al., 2014). One of the key benefits of MBTI appears to be the reduction of hyperarousal. MBTI has been found to consistently reduce self-reported physiological (e.g., sensation of heart pounding) and cognitive (e.g., racing thoughts) arousal in individuals with insomnia (Ong et al., 2008, 2009, 2014, 2018). There are theoretical justifications for reducing sleep-related arousal in individuals with insomnia, as hyperarousal has been hypothesized to be crucial in the etiology and perpetuating cycle of insomnia (Espie, 2002; Morin et al., 1993; Ong et al., 2012). Thus, reduction in both cognitive and somatic arousal may be one mechanism by which MBTI improves insomnia symptoms.

Another key symptom of insomnia theorized to be involved in its maintenance is impaired emotional functioning (Ong et al., 2012). Insomnia is characterized by significant daytime impairment and/or sleep-related distress (American Psychiatric Association 2013), including impairments in mood, such as emotional distress, mood disturbances, irritability, and emotional dysregulation (Ong et al., 2018), and sleep-related anxiety and depression (Jansson \& Linton, 2007). It is theorized that the principles of mindfulness, including increasing acceptance and awareness, and decreasing attachment, may uniquely target mood, to improve sleep outcomes (Ong et al., 2012). Currently, there is mixed evidence with regard to the impact of mindfulness-based therapies on mood in individuals with insomnia (Black et al., 2015; Garland et al., 2014; Ong et al., 2018; Yook et al., 2008; Zhang et al., 2015). Thus, there is a need to further clarify the relationship between MBTI and emotional functioning.

One of the key limitations of therapist-delivered insomnia therapy is the scarcity of trained practitioners (Meaklim et al. 2020), thus hampering dissemination and implementation of the therapy. Insomnia therapies can be costly and accessibility is limited, particularly for those in regional or remote areas (Luik et al., 2017). Extending MBTI to digital platforms may address the limitations of current insomnia treatments, by increasing the convenience, time efficiency (Griffiths et al., 2006), and scalability of treatment. The efficacy of delivering digital MBTI has not been evaluated previously (Ong \& Moore, 2020; Shallcross et al., 2019). Given the high prevalence and negative implications of insomnia, broadening the population accessing treatment is invaluable.

Therefore, the current study aimed to assess the feasibility and efficacy of a digital MBTI (dMBTI) program for reducing insomnia symptoms as the primary outcome, and reducing pre-sleep arousal and improving mood as secondary outcomes, in individuals experiencing symptoms of insomnia. It was hypothesized that participants who received a 6-week dMBTI program would have significantly better outcomes on both primary and secondary outcomes compared to those assigned to a waitlist condition.

\section{Methods}

\section{Participants}

The participants were recruited via an online advertisement posted to a research portal for staff and students at 
the university and flyers posted around the campus. Flyers were also posted to community centers (e.g., local libraries). Advertising material informed the potential participant that participants were also recruited from the waiting list at the university healthy sleep clinic. The wait time for new appointments was approximately 4-6 weeks, so potential participants were asked whether they would be happy to delay their clinic appointment while completing the trial or they could withdraw from the study at any time should they wish to take up their appointment sooner. Participants were given further information about the study's details in the explanatory statement via email. Inclusion criteria were sub-threshold to clinical insomnia (as determined by an ISI score $\geq 8$ ), complaints of sleep disturbances at least three nights a week for at least 1 month, fluent in English, and at least 18 years old. Exclusion criteria were a current or history of psychiatric conditions with the exception of treated depressive and anxiety disorders, other sleep disorders (including high risk of sleep apnea on the STOPBANG Questionnaire) unless medically treated, medical conditions or medications that may affect sleep, and learning disabilities.

A priori sample size was calculated based on the primary outcome (ISI), using summary data from Ong et al. (2014), comparing an 8-week MBTI to a self-monitoring control; this study found that MBTI reported lower scores on ISI compared to control, with a Cohen's d of 1.02. It was estimated that 34 participants were required to detect an effect size of 1.02 between groups on the ISI, with $80 \%$ power and $\alpha$ of 0.05 , using a two-tailed test.

The consort diagram of the flow of participants is presented in Fig. 1. Thirty-two participants were initially recruited and randomly allocated to either treatment or waitlist groups. Five participants withdrew from the study after randomization; one was withdrawn prior to commencement of the program, and four withdrew during the study, leaving a final sample of 27. All participants who withdrew had a moderate level of insomnia at baseline, except for one who had a severe insomnia at baseline.

The means, standard deviations, and baseline insomnia scores of the sample are displayed in Table 1. The final sample included 26 females and one male, aged between 19 and 60 years $(M=29.44$ years, $S D=11.97$ years). There were 14 participants in the intervention group and 13 in the waitlist group; the groups were well balanced on age, and there were no significant differences on any of the baseline measures between group. The majority of participants had moderatesevere insomnia at baseline, with three participants reporting sub-threshold insomnia.

\section{Procedure}

This study was a randomized waitlist control trial of an online MBTI intervention compared to a waitlist control condition with a 1:1 allocation ratio, utilizing a two (group) by three (time) repeated measures design. Ethics approval was obtained from Monash University Human Research Ethics Committee prior to the study. The study was registered on the Australian and New Zealand Clinical Trials Registry (ACTRN12620000398909). Eligibility was determined by screening questionnaires, including the STOP-BANG and ISI during a subsequent telephone interview, and written informed consent was obtained from all participants. All participants completed the ISI, PSAS, and PANAS at baseline via Qualtrics. Participants were then randomly allocated to either the intervention or waitlist groups, via
Fig. 1 Consort diagram of flow of participants

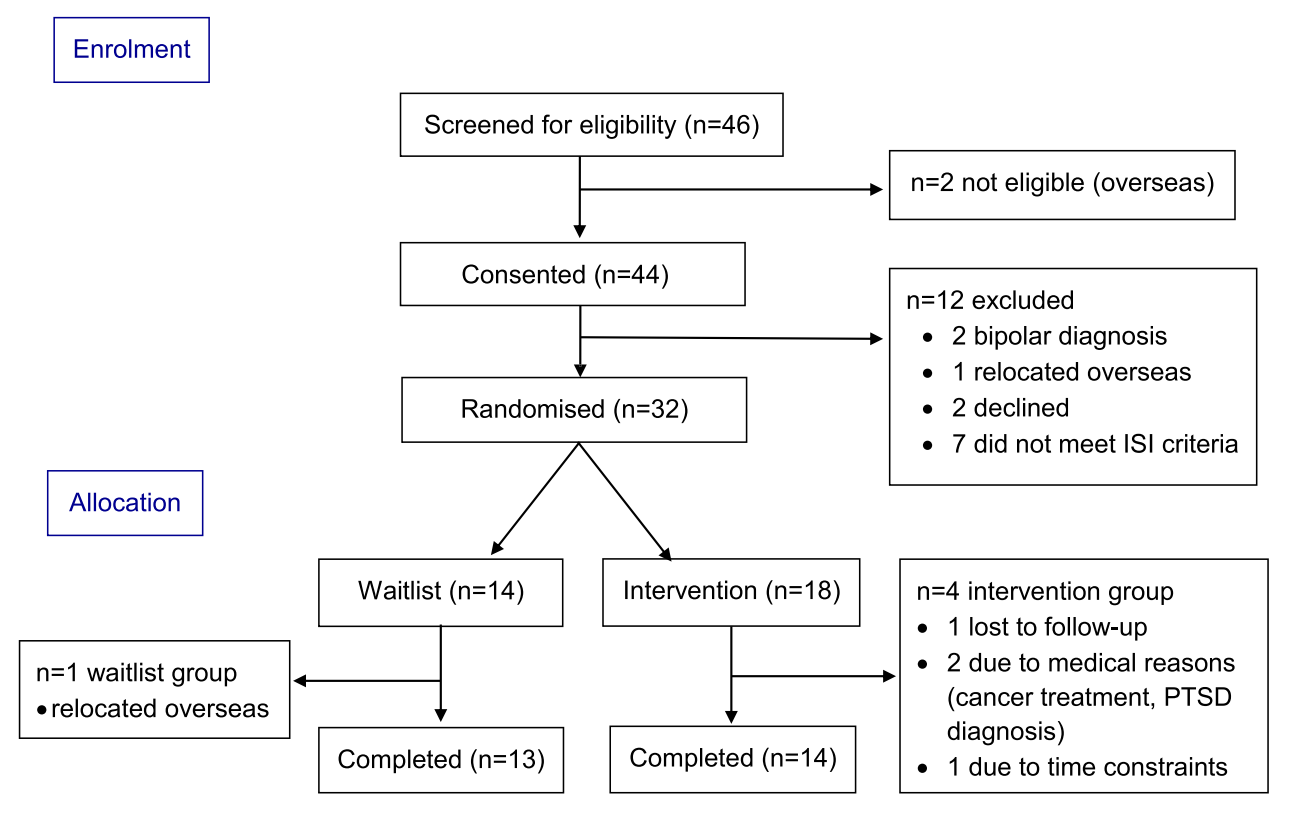


Table 1 Descriptive statistics of the sample and outcome measures at baseline $(n=27)$

\begin{tabular}{lll}
\hline Participant characteristic & Treatment group & Waitlist group \\
\hline$N$ & 14 & 13 \\
Age (years) & $27.6(10.42)$ & $31.5(13.57)$ \\
Mean (SD) & $20-59$ & $19-60$ \\
Range & $14: 0$ & $12: 1$ \\
Sex (female:male) & & \\
Insomnia severity category at & & 1 \\
$\quad$ baseline & 2 & 12 \\
Sub-threshold (ISI <10) & 12 & $16.77(3.42)$ \\
Clinical (ISI 10-28) & $16.29(4.69)$ & $13.85(4.86)$ \\
Baseline ISI & & $24.46(8.78)$ \\
Baseline PSAS & $17.07(8.08)$ & \\
Somatic subscale & $27.86(8.13)$ & $26.38(7.19)$ \\
Cognitive subscale & & $21.85(7.61)$ \\
Baseline PANAS & $29.14(8.72)$ & \\
Positive affect & $23.07(10.03)$ & \\
Negative affect &
\end{tabular}

Note. $S D$ standard deviation; $\alpha=$ Cronbach's alpha, ISI insomnia severity index, PANAS positive affect negative affect schedule, PSAS pre-sleep arousal scale

random number generation using block randomization. Participants were not blinded to group allocation. The intervention group accessed the program online via a link, and asked to complete one module of the program per week, for 6 weeks. Waitlist participants were asked to not commence any insomnia treatment during the study and were given free access to the program post-study. Participants were also asked to refrain from completing any other treatment during the study. Participants were provided a link to the Qualtrics survey to complete at the end of module three/week 3 (mid) and at the end of the study (week 6; post). The KIMS was delivered at the end of treatment. Recruitment commenced in April 2019, and data was collected from May 2019 and June 2020. Participants in the intervention group completed an exit interview at the conclusion of the study, and program usage data was obtained from A Mindful Way. Participants received a \$A50 gift voucher upon completion.

\section{Intervention}

The intervention was a 6-week online MBTI program $A$ Mindful Way to Healthy Sleep (amindfulway.com.au). The program comprised of six, weekly online modules, which included video lessons, written content, a course workbook for reflective exercises, downloadable meditation recordings, and a weekly quiz. The mindfulness training followed the teaching structure, similar to the MBTI program developed by Ong et al. (2008), which included "informal" mindfulness practices and "formal" foundational mindfulness practices (breath awareness, body scan, mindful movement, 3-min breathing space, and mindfulness of thoughts and emotions). Daily meditation practice of $15-30 \mathrm{~min}$ was expected. The CBTi training included sleep hygiene, stimulus control, sleep restriction, cognitive restructuring, and relaxation training. Participants logged into the A Mindful Way website each week and completed the training in their own time. The program involved automated email reminders (and encouragement provided in these emails) provided to participants throughout the course, and a psychologist responded to any non-clinical questions (e.g., technical issues).

\section{Measures}

Feasibility was assessed across four domains of the framework for conceptualizing feasibility studies developed by Bowen et al. (2009). Acceptability was assessed via selfreport of the overall program satisfaction and perceived benefit during exit interviews conducted in the intervention group. Implementation was assessed by program adherence (defined as the percentage of modules completed) and study attrition rates. Practicality was assessed via responses to weekly questionnaires that collected qualitative information after the completion of each weekly module (e.g., "What were the barriers (if any) to putting what you learned into practice?"). Preliminary efficacy was operationalized as effect size estimations of changes in primary and secondary outcomes in the intervention vs waitlist groups.

The primary outcome was symptoms of insomnia, measured using the insomnia severity index (ISI), a 7-item selfreport questionnaire that measures the severity of insomnia symptoms in the last 2 weeks (Bastien et al., 2001). This scale was also used as a screening tool for study inclusion. An example item is "How satisfied/dissatisfied are you with your current sleep pattern?". Greater total scores indicate higher clinical risk for insomnia. Measured on a 5-point Likert scale, the first three questions range from zero "none" to four "very severe," and questions four to seven range from zero "very satisfied" to four "very dissatisfied." All items were summed to obtain a total score ranging from 0 to 28 , and indicate sub-threshold insomnia (8-14), clinical insomnia with moderate severity (15-21), and severe clinical insomnia (22-28) (Bastien et al., 2001). Total scores $\geq 10$ have been found to be optimal for detecting insomnia cases in community samples (Morin et al., 2011), and therefore this was used as the cut-off for clinical cases in the current study. Remission was defined as a score $<10$ on the ISI posttreatment. A change of $\geq 8$ points on the ISI from pre-to post-study was considered a clinically significant response (Morin et al., 2011). The ISI had good internal reliability in this study $(\alpha=0.70)$.

The pre-sleep arousal scale (PSAS), a 16-item self-report questionnaire, measures the subjective intensity of arousal 
experienced when attempting to fall asleep on two subscales: somatic arousal and cognitive arousal (Nicassio et al., 1985). The PSAS is measured on a 5-point Likert scale; responses range from one "not at all" to five "extremely." Higher total scores indicate increased pre-sleep arousal. An example item is "worrying about falling asleep." The cognitive $(\alpha=0.93)$ and somatic $(\alpha=0.90)$ subscales had excellent internal reliability in the current study.

The PANAS is a 20-item self-report questionnaire that measures mood over the past week (Watson et al., 1988). It has two subscales: positive affect (PA) and negative affect (NA). The PANAS is measured on a 5-point Likert scale, ranging from one "very little/not at all" to five "extremely," and is scored by summing all items on each scale, with a total maximum score of 50 for each subscale. An example item is "indicate the extent you have felt excited over the past week." Higher total scores for PA items indicate higher PA, and lower scores on the NA scale indicate lower NA. The PA $(\alpha=0.90)$ and NA $(\alpha=0.90)$ subscales had excellent internal reliability in this study.

The Kentucky Inventory of Mindfulness Skills (KIMS) is a 39-item self-report measure of mindfulness skills (Baer et al., 2004). The KIMS is measured on a 5-point Likert scale; responses range from zero "never or very rarely true" to five "very often or always true," and scored by summing each subscale (observe, describe, act with awareness, accept without judgment), with some items reversed scored.

\section{Data Analyses}

Implementation of the program was assessed using the following outcomes: attrition (drop outs prior to completion) across the whole sample (\%), attrition in the intervention group (\%), and module completion (\%). Acceptability and practicality were assessed using overall themes identified from weekly questionnaires and the end of study interview in the intervention group. Preliminary efficacy was assessed using Cohen's $d$ to determine the effect sizes of the differences between the groups at mid- and post-study.

Descriptive statistic of means and standard errors were calculated for each outcome across all time points. Three participants had missing data for their entire mid-point questionnaires; for this reason, the primary analyses included pre- and post-intervention data only. A repeated measures analysis of variance (ANOVA) was conducted to examine the main effects of group (intervention and waitlist control) and time (baseline and post-study) and their interaction, on all three outcomes of ISI scores, PSAS (somatic and cognitive subscales), and PANAS (PA and NA subscales). The effect sizes were calculated using partial eta square. For participants who had clinical insomnia (ISI $\geq 10$ ) at baseline, response and remission rates were calculated. For response rate, a clinically significant ISI change score was defined as reduction of 8 points or more on the ISI (Morin et al., 2011), and remission rate was calculated as the proportion of participants who fell below an ISI of 10 post-study in each group. Within group paired samples, $t$ tests were employed to determine if there were any statistically significant withintreatment effects in the outcome measures at each time point. An independent samples $t$ test was performed to compare the KIMS scores in the treatment versus the waitlist groups at the end of the study. One participant in the treatment group did not complete the KIMS questionnaire, leaving $N=26$ for this analysis. The significance for $p$ value was set at 0.05 for all analyses.

\section{Results}

\section{Program Feasibility}

\section{Acceptability}

Overall, the course was positively received and accepted, with participants reporting that they were "more relaxed about not sleeping" and "slept better throughout the course." The participants also reported that they were overall "satisfied" with the program and that they had found it "helpful."

\section{Implementation}

The overall attrition rate of the study was $16 \%$, with five out of the initial 32 participants randomized subsequently withdrawing from the study. All participants randomized to the waitlist group, except one, completed the trial. Of the 18 participants randomized into the intervention group, 14 completed the protocol (study completion rate $=78 \%$ ); three participants dropped out, and one was withdrawn from the study by the investigators due to medical reasons. The average program completion rate of the six modules was $79 \%$.

\section{Practicality}

The barriers identified included the feasibility of scheduling time to practice the program and fitting sleep strategies into everyday life.

\section{Preliminary Efficacy}

Means standard deviations and Cohen's $d$ effect sizes for difference in primary and secondary outcomes between groups at mid- and post-study are shown in Table 2. Differences were observed between groups at post-study for 
the ISI and PSAS subscales, with moderate to large effect sizes. Smaller effect sizes were noted for the PANAS positive affect scale, with negligible effects for negative affect. Further inferential statistics were also conducted on these outcomes.

\section{The Efficacy of the Program on the Severity of Insomnia Symptoms}

The results of the repeated measures ANOVA for the ISI are reported in Table 3. There was a significant interaction effect between group and time on the ISI, with the treatment group demonstrating a significantly reduced severity of insomnia symptoms at follow-up compared to the waitlist group $(p<0.001$, Fig. 2). There was also a significant main effect of group $(p=0.014)$ and time $(p<0.001)$, with an overall reduction in the ISI over the study (Fig. 2).

Within the treatment group, there was a mean 9.4-point reduction in the ISI score, from 16.29 at baseline to 6.86 post-study, representing a clinically significant change (Morin et al., 2011). Of the participants who had an ISI $\geq 10$ at baseline, 9 of 12 participants in the intervention group achieved remission (75\%), and 1 of 12 participants (8.3\%) in the waitlist group achieved remission, scoring an ISI below 10 post-study. The response rate in the intervention group was $83 \%$, with 10 out of 12 participants having a clinically significant reduction in ISI score $(\geq 8)$, compared to 1 of 12 participants $(8.3 \%)$ in the waitlist group.

\section{The Efficacy of the Program on Pre-sleep Arousal}

The results of the repeated measures ANOVA for the PSAS are reported in Table 3. There were significant interaction effects between group and time for both the cognitive $(p=0.03)$ and somatic $(p=0.02)$ subscales of the PSAS (Fig. 3 and 4$)$. There
Table 3 Main and interaction effects from the repeated measures ANOVA on insomnia symptoms, pre-sleep arousal, and affect

\begin{tabular}{|c|c|c|c|c|c|}
\hline Variable & $d f$ & $M S$ & $F$ & $p$ & Partial Eta ${ }^{2}$ \\
\hline \multicolumn{6}{|l|}{ ISI } \\
\hline Group & 1 & 224.68 & 6.93 & $0.014 *$ & .22 \\
\hline Time & 1 & 458.17 & 44.77 & $<.001 *$ & .64 \\
\hline Time $\times$ group & 25 & 174.61 & 17.06 & $<.001 *$ & .41 \\
\hline \multicolumn{6}{|c|}{ PSAS cognitive scale } \\
\hline Group & 1 & 3.83 & 0.03 & .86 & .001 \\
\hline Time & 1 & 847.48 & 21.73 & $<.001 *$ & .47 \\
\hline Time $\times$ group & 25 & 208.07 & 5.34 & $.03 *$ & .48 \\
\hline \multicolumn{6}{|c|}{ PSAS somatic scale } \\
\hline Group & 1 & 1.23 & .02 & .89 & .001 \\
\hline Time & 1 & 15.64 & .80 & $.38^{*}$ & .03 \\
\hline Time $\times$ group & 25 & 115.19 & 5.89 & $.02 *$ & .19 \\
\hline \multicolumn{6}{|l|}{ PANAS PA } \\
\hline Group & 1 & 212.75 & 2.38 & .14 & .09 \\
\hline Time & 1 & 8.32 & .18 & .68 & .01 \\
\hline Time $\times$ group & 25 & 19.88 & .42 & .52 & .02 \\
\hline \multicolumn{6}{|l|}{ PANAS NA } \\
\hline Group & 1 & 91.64 & 3.15 & .88 & .11 \\
\hline Time & 1 & 423.86 & 14.57 & $.001 *$ & .37 \\
\hline Time $\times$ group & 25 & 91.64 & 3.15 & .09 & .11 \\
\hline
\end{tabular}

Note. $N=27 . M S$ mean square. ${ }^{*} p<.05$

$I S I$ insomnia severity index, PANAS positive affect negative affect schedule, PSAS pre-sleep arousal scale

was also a significant main effect of time for cognitive arousal $(p<0.001)$, but not for somatic arousal $(p=0.38)$.

\section{The Efficacy of the Program on Daily Affect}

The results of the repeated measures ANOVA for the PANAS subscales are reported in Table 3. There were no
Table 2 Descriptive statistics and effect sizes for difference in ISI, PSAS, and PANAS between groups at mid (3 weeks) and post-study

\begin{tabular}{lllll}
\hline Outcome & Time point & $\begin{array}{l}\text { Intervention } \\
\text { Mean (SD) }\end{array}$ & $\begin{array}{l}\text { Waitlist } \\
\text { Mean (SD) }\end{array}$ & Cohen's $d$ \\
\hline ISI & Mid-study & $11.14(3.35)$ & $14.30(5.52)$ & -.72 \\
& Post-study & $6.86(3.63)$ & $14.60(6.87)$ & 1.49 \\
Cognitive arousal (PSAS) & Mid-study & $22.36(8.83)$ & $20.60(10.18)$ & .19 \\
& Post-study & $16.00(7.10)$ & $19.20(11.48)$ & -.35 \\
Somatic arousal (PSAS) & Mid-study & $14.57(4.07)$ & $14.20(4.41)$ & .08 \\
& Post-study & $13.07(4.98)$ & $14.40(6.31)$ & -.24 \\
Positive affect (PANAS) & Mid-study & $24.21(9.90)$ & $23.70(11.25)$ & .05 \\
& Post-study & $29.57(7.47)$ & $24.80(10.84)$ & .53 \\
Negative affect (PANAS) & Mid-study & $18.79(6.20)$ & $19.10(6.62)$ & -.05 \\
& Post-study & $14.86(5.78)$ & $16.20(6.00)$ & -.02 \\
\hline
\end{tabular}

Note. Mid-study: $n=14$ in the treatment group, $n=10$ in the waitlist group. Post-study: $n=14$ in the treatment group, $n=13$ in the waitlist group. ISI insomnia severity index, PANAS positive affect negative affect schedule, PSAS pre-sleep arousal scale 
Fig. 2 Insomnia severity index scores in the treatment and waitlist groups at baseline and post-study. Note: Error bars represent $95 \%$ CI
Fig. 3 Cognitive subscale scores from the PSAS in the treatment and waitlist groups at baseline and post-study. Note: Error bars represent 95\% CI
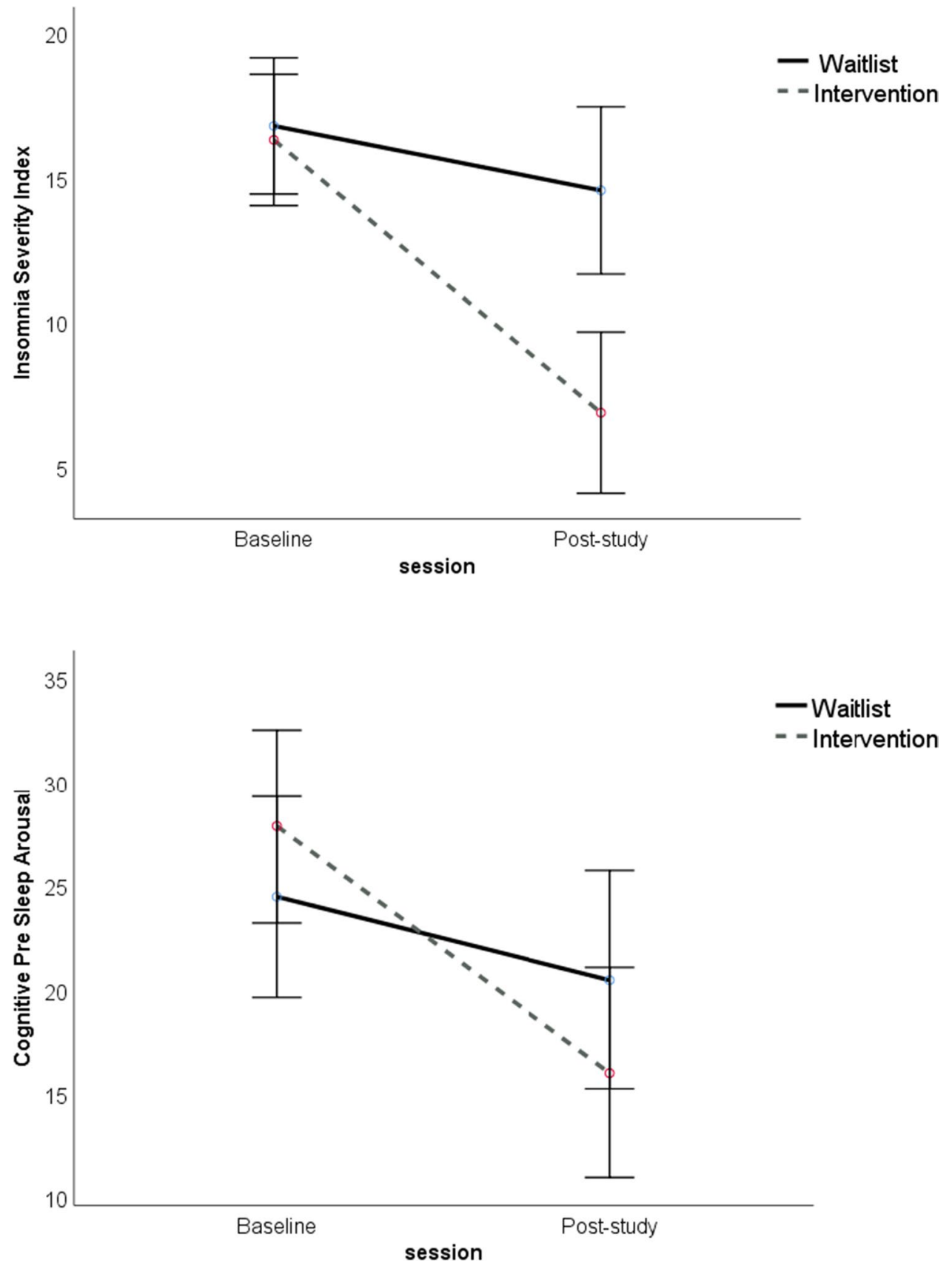

significant interaction effects between group and time for either subscale of the PANAS (Fig. 5 and 6). There was, however, a significant main effect of time, with negative affect improving in both groups at follow-up $(p=0.001$; Fig. 5).

\section{Mindfulness Skills}

There was no significant difference observed in KIMS scores at follow-up between the treatment group $(\mathrm{M}=129.77$, $\mathrm{SD}=13.24)$ and the waitlist group $(\mathrm{M}=121.62, \mathrm{SD}=21.04)(t$ $(24)=-1.182, p=0.25 ; 95 \%$ CI [-22.39, 6.08], Cohen's $d=0.46)$.

\section{Discussion}

This study examined the feasibility and efficacy of a dMBTI program for improving sleep in individuals with sub-clinical to clinical levels of insomnia. Following this novel dMBTI program, the treatment group revealed significant improvements in the severity of insomnia symptoms and in pre-sleep arousal compared to the waitlist group; however, there were no significant improvements in daily affect. It is the first study to our knowledge to investigate the feasibility and efficacy of an dMBTI program in individuals experiencing symptoms of insomnia 
Fig. 4 Somatic Subscale scores from the PSAS in the treatment and waitlist groups at baseline and post-study. Note: Error bars represent $95 \% \mathrm{CI}$
Fig. 5 Positive affect subscale from the PANAS in the treatment and waitlist groups at baseline and post-study. Note: Error bars represent 95\% CI
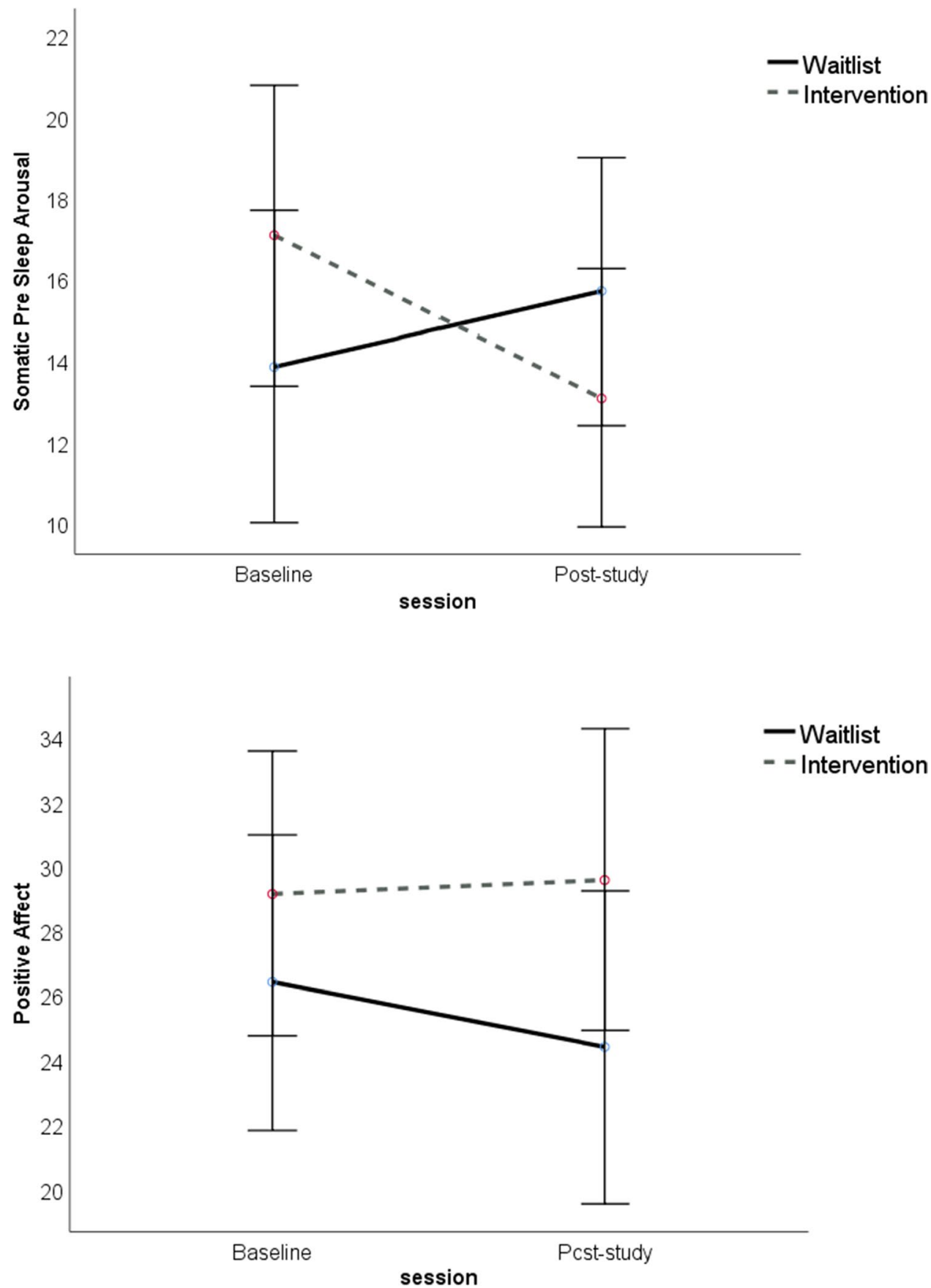

and adds to the growing body of literature demonstrating the benefits of digital therapies for insomnia (Grierson et al., 2020).

The first aim of this study was to assess the feasibility, acceptability, and barriers of this dMBTI program. Participants reported positive feedback from the program including promoting relaxed attitudes around sleep and improved sleep. This is comparable with other online insomnia programs (Kuhn et al., 2016). Time constraints and the feasibility of implementing sleep-strategies were identified as barriers by participants in the intervention group. Participants were concerned about the feasibility of scheduling the time to practice the program, which included 1- to 2-h weekly modules and the practice of mindfulness exercises, and fitting sleep strategies into everyday life. Despite this, we demonstrated a $79 \%$ completion rate in this study. Other online insomnia treatments have identified similar barriers, including themes of competing demands in an online CBTi program (Hermes et al., 2019). The similar profile of positive perceptions and barriers identified in this program 
Fig. 6 Means and standard errors of the negative affect subscale of the PANAS in the treatment and waitlist groups at baseline and post-study. Note: Error bars represent 95\% CI

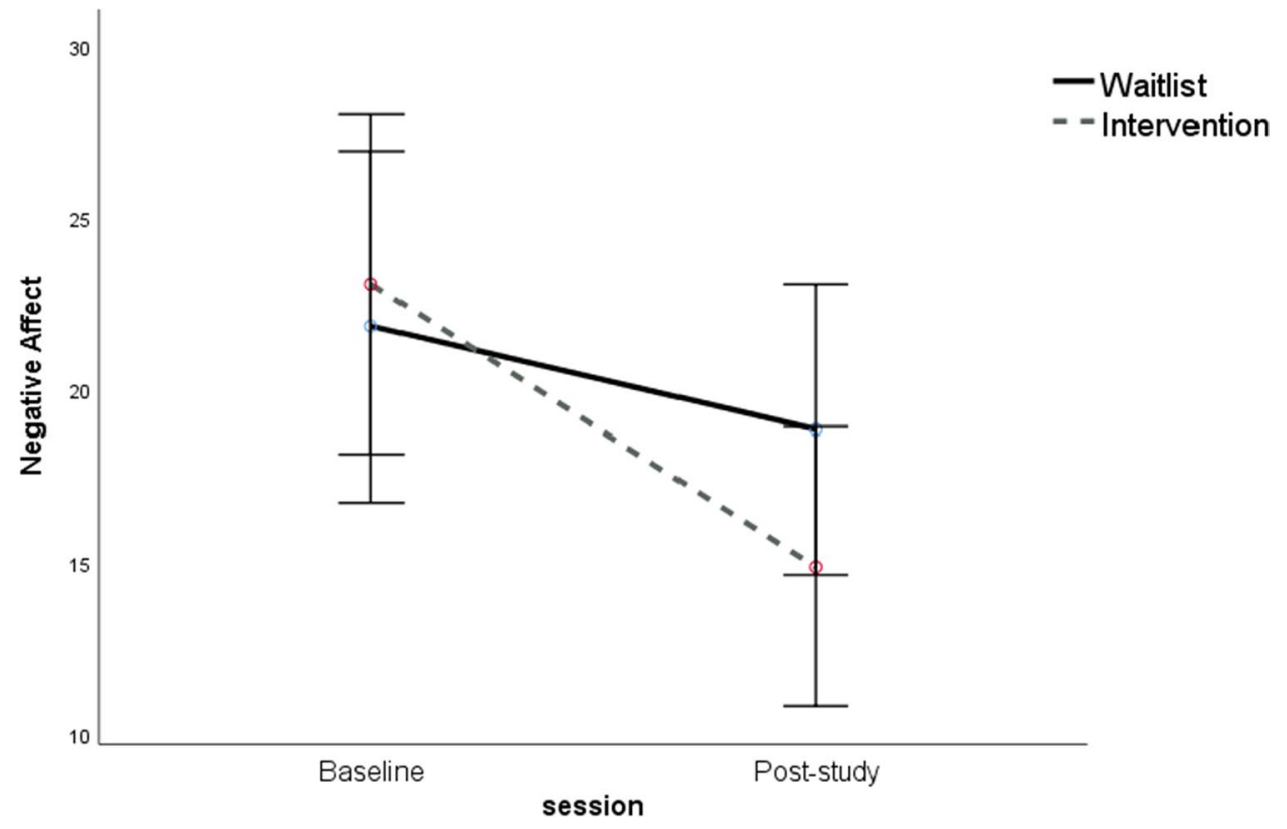

together with those in other online insomnia interventions would suggest that the current intervention's feasibility may be comparable to that of other online insomnia interventions.

In this study, the program module completion rate was $79 \%$; this is higher treatment adherence reporting for other online insomnia treatments, estimated at $52 \%$ in a metaanalysis (Horsch et al., 2015). Although there is currently no standard in adherence measures, definitions include interaction with the program (e.g., model completion), drop-out rates, and likeliness to adhere to the treatment post-experiment (Horsch et al., 2015). In comparison to insomnia interventions delivered face-to-face, which have identified attrition as a "major issue" (Hermes et al., 2019), the current study also reported better retention, with a drop-out rate of $22 \%$ in the intervention group. A meta-analysis reported that therapist-delivered CBTi had drop-out rates of up to 33\% (Okajima et al., 2011). The lower attrition rates and increased adherence observed in this study may be explained by the extra support offered as part of the A Mindful Way program, whereby a clinical psychologist and the researchers responded to the participants' questions and provided email reminders and encouragement. Previous research has found that this type of support is important for CBTi program adherence (Andersson, 2009). Ultimately, the current study's good completion rate would further suggest that dMBTI may be a feasible treatment for insomnia.

The results of this study supported the hypothesis that a 6-week dMBTI program would reduce insomnia symptoms, as a significantly greater reduction in ISI scores was observed in the treatment compared to the control group. Moreover, a clinically significant ISI change score of 9.43 was observed in the intervention group (Morin et al., 2011).
In comparison to other insomnia treatments, a meta-analysis reported a change score of 5.88 on the ISI for dCBTi (Ye et al., 2016), indicating that dMBTI may be comparable to other online insomnia interventions. Furthermore, of the participants who had clinical levels of insomnia at baseline, $75 \%$ achieved remission (i.e., ISI score < 10) after the intervention. There was also a response rate of $85 \%$ in the intervention group, compared to only $8 \%$ in the waitlist group, indicating a moderate improvement. This is comparable to an in-person MBTI trial, which observed a remission rate of $50 \%$ and response rate of $78 \%$ in a sample of individuals with chronic insomnia (Ong et al., 2014). While these figures should be taken with caution given the small sample size, the promising remission and response rates observed in this study may suggest that dMBTI could be an effective clinical treatment.

The significant reduction in ISI scores in the treatment versus control group is consistent with previous research demonstrating ISI improved following group-delivered MBTI (Ong et al., 2008, 2009, 2014). For example, the average treatment response of MBTI on the ISI was reported as 4.56 in one controlled study (Ong et al., 2014) and 5.33 in an uncontrolled study (Ong et al., 2008). While previous research demonstrated these improvements in the severity of insomnia symptoms when MBTI was administered faceto-face, the current study is the first to evaluate dMBTI. The current study highlights that MBTI delivered through a digital format can improve the severity of insomnia symptoms beyond face-to-face delivery. Additionally, this study employed a novel approach to reducing insomnia symptoms by delivering the intervention individually, in contrast to previous research that delivered MBTI in a group format. 
Moreover, individually delivered programs can help facilitate increased autonomy in an individual's treatment of their insomnia. The significant reduction in ISI scores found in this study not only aligns with existing literature, but also extends MBTI to novel delivery platforms, thus supporting $\mathrm{dMBTI}$ as a viable treatment for significantly reducing the severity of insomnia symptoms.

The second hypothesis, that a 6-week dMBTI program would reduce pre-sleep arousal, as measured on somatic and cognitive subscales of the PSAS, compared to waitlist controls, was supported. This finding supports the theory that arousal is a pivotal mechanism in the maintenance of insomnia and that insomnia symptoms can be improved by reducing arousal (Espie, 2002; Morin et al., 1993; Ong et al., 2012). These significant results align with the majority of previous research, which has consistently demonstrated that MBTI reduced arousal (Ong et al., 2014, 2018, 2008, 2009). Furthermore, the finding that the cognitive subscale (pre $=27.86$, post $=16.00)$ had a greater reduction than the somatic subscale (pre $=17.07$, post $=13.07$ ) in the treatment group supports previous findings of a greater reduction in the cognitive compared to the somatic subscale after group MBTI (Ong et al., 2008). Thus, the current study demonstrates support for the efficacy of dMBTI for reducing arousal and may help to explain why MBTI may be superior to CBTi for some individuals.

An exploratory aim of this study was to examine the impact of dMBTI on affect, as measured by the PANAS. There was no significant interaction observed for either positive or negative affect. A main effect of time was found for $\mathrm{NA}$, with both groups showing an improvement in negative affect across the trial. Improvements in mood in individuals with insomnia have been observed in previous studies which have utilized more general mindfulness-based therapies (not specifically targeting insomnia) and different measures of mood (e.g., depression, stress, anxiety) (Black et al., 2015; Garland et al., 2014; Yook et al., 2008). Ong et al. (2018) proposed that these general mindfulness programs might have a greater effect on mood than MBTI programs that are specifically for insomnia; this may explain the null results for affect in the present study. It is also possible that our study was not sufficiently powered to detect changes in affect, given the small effect sizes observed. We also excluded individuals with psychiatric disorders, including untreated anxiety and depression; therefore, our participants did not have any significant mood disturbance at baseline. Ultimately, the non-significant results for daily affect observed in the present study highlight the need for additional research to clarify the relationship between mood and MBTI.

Interestingly, the present study revealed no significant difference in KIMS scores in the treatment versus waitlist group at follow-up. This aligns with previous finding of a non-significant within-group improvement on the KIMS after a 6-week MBTI program or at a 12-month follow-up (Ong et al., 2009). These pilot studies did, however, observe a negative correlation between the amount of mindfulness meditations undertaken and hyperarousal, suggesting that mindfulness may assist with specific aspects of insomnia (e.g., sleep-related arousal). The lack of improvement in mindfulness skills in the current study may lead one to conclude that the positive benefits on sleep observed in the intervention group may be primarily driven by the CBTi components of the MBTI program. The current design precluded us from exploring the specific effects of meditation compared to the components of CBTi; this would be an important avenue for future studies. A limitation of the current study was that KIMS was only assessed at follow-up; therefore, we do not know what baseline levels of mindfulness were present in our groups and whether any change occurred within-subjects over the duration of the study. There are also some limitations to self-report measures of mindfulness (e.g., Park et al. 2013). Nevertheless, it is possible that a 6-week period may not allow participants sufficient exposure to mindfulness to be able to fully integrate mindfulness into everyday life, with flow-on effects for arousal and sleep. Whether these skills develop further over time need to be evaluated in longer intervention studies, as well as assessing how the development of mindfulness skills map on to improvements in insomnia and other symptoms.

\section{Limitations and Future Directions}

In addition to the clinical population recruited, this study also included three individuals with sub-clinical symptoms (ISI < 10). The results from this pilot study indicate that dMBTI may be an appropriate and efficacious treatment option for individuals with sub-clinical insomnia, who may be less inclined to access face-to-face treatment for their sleep. Future research is needed to investigate whether there are similar improvements found between individuals with sub-clinical versus moderate insomnia; this was not possible in the current pilot due to the limited sample size. Although large effect sizes were observed in the treatment group for the ISI, the study may have been underpowered to detect significant improvements in other aspects of sleep and mood, given the small sample size. Additionally, this study only assessed outcomes over a six-week protocol; only one previous MBTI study included a 12-month follow-up, demonstrating ongoing benefits of MBTI on sleep outcomes (Ong et al., 2009). Future studies should also determine whether the benefits of dMBTI reducing ISI scores and sleep-related arousal are sustained in the long term. An inherent limitation of waitlist control designs is that they do not allow for comparison of different treatments; thus, it is possible that the outcomes may reflect a general treatment effect. 
Mindfulness-based interventions have a high risk of expectation bias, and participants may show purported intervention effects which could have resulted from non-specific factors such as quality of therapeutic alliance and relationship, empathy, and being non-judgmental. It will be important for future studies to compare MBTI with an active control, or with $\mathrm{CBTi}$, in order to better understand the mechanisms underlying improvement in sleep outcomes. Finally, as the sample primarily consisted of females (only one male completed the trial), generalizing the findings to males with insomnia is not possible. We excluded individuals who were at high risk of sleep apnea; therefore, this may have excluded a number of potential participants who were male. Since females form the greater majority of the general insomnia population (Zhang \& Wing, 2006), the current results are generalizable to this demographic. In light of the above limitations, prospective studies utilizing larger and more diverse samples are needed to further validate the results.

The present findings add to the growing body of literature supporting the efficacy of mindfulness-based therapies for insomnia. dMBTI may present an enhanced treatment option to CBTi, which is important given that CBTi is not suitable or effective for everyone (Shallcross et al., 2019). This study also has theoretical implications for understanding the mechanism of insomnia. Arousal has been theorized to be involved in the etiology of insomnia, and mood has been suggested to be implicated in this processes (Ong et al., 2012). In contrast, the non-significant findings for mood in this study suggest that further clarification is needed to understand the role of emotional functioning in the theoretical underpinnings of insomnia. Furthermore, this study's innovative feature of assessing dMBTI extends the existing literature base beyond face-to-face delivery alone. This has novel research implications by demonstrating that MBTI can also be efficacious via online platforms. The extension of MBTI to online platforms can also have widespread clinical implications by increasing the scalability and accessibility of the intervention, reducing individual and health service costs, decreasing isolation, and increasing convenience and time efficiency (Griffiths et al., 2006), all of which are particularly critical during this time of the COVID-19 pandemic. This would allow a wider population to access treatment, which, given the high prevalence and negative implications of insomnia (Qaseem et al., 2016), is invaluable.

Acknowledgements The authors wish to thank Dr. Giselle Withers from A Mindful Way for providing access to the program to our participants.

Author Contribution LK, MJ, and BB: designed this research project and gained ethics approval. LK: conducted the recruitment process with the assistance of Monash Healthy Sleep Clinic. The author screened the participants, reviewed the literature, collected and scored the data, conducted the data analysis, and wrote the manuscript. MJ and BB: collaborated in writing and the editing of the manuscript and helped guide the data analysis. All authors have seen and approved the manuscript.

Data Availability De-identified data will be made available upon written request to the corresponding author.

\section{Declarations}

Ethics Approval Ethics approval was obtained from Monash University Human Research Ethics Committee (project ID: 18774).

Informed Consent Written informed consent was obtained from all participants prior to undertaking the study.

Conflict of Interest The research was supported by A Mindful Way Training, who provided access to the program for research participants. The authors have no other conflicts of interest to declare.

Research performed at Monash University, Melbourne, Australia.

\section{References}

Adams, R. J., Appleton, S. L., Taylor, A. W., Gill, T. K., Lang, C., McEvoy, R. D., \& Antic, N. A. (2017). Sleep health of Australian adults in 2016: results of the 2016 sleep health foundation national survey. Sleep Health, 3(1), 35-42. https://doi.org/10.1016/j.sleh. 2016.11.005

American Psychiatric Association. (2013). Diagnostic and statistical manual of mental disorders: DSM-5 (5th ed.). https://doi.org/10. 1176/appi.books.9780890425596

Andersson, G. (2009). Using the Internet to provide cognitive behaviour therapy. Behaviour Research and Therapy, 47(3), 175-180. https://doi.org/10.1016/j.brat.2009.01.010

Baer, R. A., Smith, G. T., \& Allen, K. B. (2004). Assessment of mindfulness by self-report: The Kentucky Inventory of Mindfulness Skills. Assessment, 11(3), 191-206. https://doi.org/10.1177/10731 91104268029

Bastien, C. H., Vallières, A., \& Morin, C. M. (2001). Validation of the Insomnia Severity Index as an outcome measure for insomnia research. Sleep Medicine, 2(4), 297-307. https://doi.org/10.1016/ S1389-9457(00)00065-4

Black, D. S., O’Reilly, G. A., Olmstead, R., Breen, E. C., \& Irwin, M. R. (2015). Mindfulness meditation and improvement in sleep quality and daytime impairment among older adults with sleep disturbances: A randomized clinical trial. JAMA Internal Medicine, 175(4), 494-501.

Bowen, D. J., Kreuter, M., Spring, B., Cofta-Woerpel, L., Linnan, L., Weiner, D., Bakken, S., Kaplan, C. P., Squiers, L., Fabrizio, C., \& Fernandez, M. (2009). How we design feasibility studies. American Journal of Preventive Medicine, 36(5), 452-457. https://doi. org/10.1016/j.amepre.2009.02.002

Chung, K.-F., Yeung, W.-F., Ho, F. Y.-Y., Yung, K.-P., Yu, Y.-M., \& Kwok, C.-W. (2015). Cross-cultural and comparative epidemiology of insomnia: the Diagnostic and Statistical Manual (DSM), International Classification of Diseases (ICD) and International Classification of Sleep Disorders (ICSD). Sleep Medicine, 16(4), 477-482. https://doi.org/10.1016/j.sleep.2014.10.018

Espie, C. A. (2002). Insomnia: Conceptual issues in the development, persistence, and treatment of sleep disorder in adults. Annual Review of Psychology, 53(1), 215-243.

Garland, S. N., Carlson, L. E., Stephens, A. J., Antle, M. C., Samuels, C., \& Campbell, T. S. (2014). Mindfulness-based stress reduction 
compared with cognitive behavioral therapy for the treatment of insomnia comorbid with cancer: A randomized, partially blinded, noninferiority trial. Journal of Clinical Oncology, 32(5), 449-457. https://doi.org/10.1200/JCO.2012.47.7265

Garland, S. N., Zhou, E. S., Gonzalez, B. D., \& Rodriguez, N. (2016). The quest for mindful sleep: A critical synthesis of the impact of mindfulness-based interventions for insomnia. Current Sleep Medicine Reports, 2(3), 142-151. https://doi.org/10.1007/ s40675-016-0050-3

Gong, H., Ni, C.-X., Liu, Y.-Z., Zhang, Y., Su, W.-J., Lian, Y.-J., Peng, W., \& Jiang, C.-L. (2016). Mindfulness meditation for insomnia: A meta-analysis of randomized controlled trials. Journal of Psychosomatic Research, 89, 1-6. https://doi.org/10.1016/j.jpsyc hores.2016.07.016

Grierson, A. B., Hobbs, M. J., \& Mason, E. C. (2020). Self-guided online cognitive behavioural therapy for insomnia: A naturalistic evaluation in patients with potential psychiatric comorbidities. Journal of Affective Disorders, 266, 305-310. https://doi.org/10. 1016/j.jad.2020.01.143

Griffiths, F., Lindenmeyer, A., Powell, J., Lowe, P., \& Thorogood, M. (2006). Why are health care interventions delivered over the internet? A systematic review of the published literature. Journal of Medical Internet Research, 8(2), e10-e10. https://doi.org/10. 2196/jmir.8.2.e10

Hermes, E. D., Merrel, J., Clayton, A., Morris, C., \& Rowe, M. (2019). Computer-based self-help therapy: A qualitative analysis of attrition. Health Informatics Journal, 25(1), 41-50. https://doi.org/10. $1177 / 1460458216683536$

Horsch, C., Lancee, J., Beun, R. J., Neerincx, M. A., \& Brinkman, W.-P. (2015). Adherence to technology-mediated insomnia treatment: A meta-analysis, interviews, and focus groups. Journal of Medical Internet Research, 17(9), e214-e214. https://doi.org/10. 2196/jmir.4115

Jansson, M., \& Linton, S. J. (2007). Psychological mechanisms in the maintenance of insomnia: Arousal, distress, and sleep-related beliefs. Behavior Research and Therapy, 45(3), 511-521.

Kalmbach, D. A., Cheng, P., Arnedt, J. T., Anderson, J. R., Roth, T., Fellman-Couture, C., Williams, R. A., \& Drake, C. L. (2019). Treating insomnia improves depression, maladaptive thinking, and hyperarousal in postmenopausal women: comparing cognitivebehavioral therapy for insomnia (CBTI), sleep restriction therapy, and sleep hygiene education. Sleep Medicine, 55, 124-134. https://doi.org/10.1016/j.sleep.2018.11.019

Kuhn, E., Weiss, B. J., Taylor, K. L., Hoffman, J. E., Ramsey, K. M., Manber, R., Gehrman, P., Crowley, J. J., Ruzek, J. I., \& Trockel, M. (2016). CBT-I coach: A description and clinician perceptions of a mobile app for cognitive behavioral therapy for insomnia. Journal of Clinical Sleep Medicine, 12(4), 597-606.

Luik, A. I., Kyle, S. D., \& Espie, C. A. (2017). Digital cognitive behavioral therapy (dCBT) for insomnia: A state-of-the-science review. Current Sleep Medicine Reports, 3(2), 48-56. https://doi.org/10. 1007/s40675-017-0065-4

Meaklim, H., Jackson, M. L., Bartlett, D., Saini, B., Falloon, K., Junge, M., Slater, J., Rehm, I. C., \& Meltzer, L. J. (2020). Sleep education for healthcare providers: Addressing deficient sleep in Australia and New Zealand. Sleep Health, 6(5), 636-650. https://doi. org/10.1016/j.sleh.2020.01.012

Morin, C. M., Belleville, G., Bélanger, L., \& Ivers, H. (2011). The insomnia severity index: psychometric indicators to detect insomnia cases and evaluate treatment response. Sleep, 34(5), 601-608. https://doi.org/10.1093/sleep/34.5.601

Morin, C., Stone, J., Trinkle, D., Mercer, J., \& Remsberg, S. (1993). Dysfuntional beliefs and attitudes about sleep among older adults with and without insomnia complaints. Psychology and Aging, 8(3), 463-467. https://doi.org/10.1037//0882-7974.8.3.463
Morin, C., Vallières, A., Guay, B., Ivers, H., Savard, J., Mérette, C., Bastien, C., \& Baillargeon, L. (2009). Cognitive behavioral therapy, singly and combined with medication, for persistent insomnia: A randomized controlled trial. JAMA, 301(19), 2005-2015. https://doi.org/10.1001/jama.2009.682

Nicassio, P. M., Mendlowitz, D. R., Fussell, J. J., \& Petras, L. (1985). The phenomenology of the pre-sleep state: The development of the pre-sleep arousal scale. Behavior Research and Therapy, 23(3), 263-271. https://doi.org/10.1016/0005-7967(85)90004-X

Okajima, I., Komada, Y., \& Inoue, Y. (2011). A meta-analysis on the treatment effectiveness of cognitive behavioral therapy for primary insomnia. Sleep and Biological Rhythms, 9(1), 24-34. https://doi.org/10.1111/j.1479-8425.2010.00481.x

Ong, J., Manber, R., Segal, Z., Xia, Y., Shapiro, S., \& Wyatt, J. K. (2014). A randomized controlled trial of mindfulness meditation for chronic insomnia. Sleep, 37(9), 1553-1563. https://doi.org/ $10.5665 /$ sleep. 4010

Ong, J., \& Moore, C. (2020). What do we really know about mindfulness and sleep health? Current Opinon Psychology, 34, 18-22. https://doi.org/10.1016/j.copsyc.2019.08.020

Ong, J., \& Smith, C. E. (2017). Using mindfulness for the treatment of insomnia. Current Sleep Medicine Reports, 3(2), 57-65.

Ong, J., Ulmer, C. S., \& Manber, R. (2012). Improving sleep with mindfulness and acceptance: A metacognitive model of insomnia. Behavior Research and Therapy, 50(11), 651-660. https://doi.org/ 10.1016/j.brat.2012.08.001

Ong, J., Xia, Y., Smith-Mason, C., \& Manber, R. (2018). A randomized controlled trial of mindfulness meditation for chronic insomnia: effects on daytime symptoms and cognitive-emotional arousal. Mindfulness, 9, 1702-1712. https://doi.org/10.1007/ s12671-018-0911-6

Ong, J. C., Shapiro, S. L., \& Manber, R. (2008). Combining mindfulness meditation with cognitive-behavior therapy for insomnia: A treatment-development study. Behavior Therapy, 39(2), 171-182. https://doi.org/10.1016/j.beth.2007.07.002

Ong, J. C., Shapiro, S. L., \& Manber, R. (2009). Mindfulness meditation and cognitive behavioral therapy for insomnia: A naturalistic 12-month follow-up. Explore (New York, N.Y.), 5(1), 30-36. https://doi.org/10.1016/j.explore.2008.10.004

Park, T., Reilly-Spong, M., \& Gross, C. R. (2013). Mindfulness: a systematic review of instruments to measure an emergent patientreported outcome (PRO). Quality of Life Research, 22(10), 26392659. https://doi.org/10.1007/s11136-013-0395-8

Qaseem, A., Kansagara, D., Forciea, M. A., Cooke, M., \& Denberg, T. D. (2016). Management of chronic insomnia disorder in adults: A clinical practice guideline from the American College of Physicians. Annals of Internal Medicine, 165(2), 125-133. https://doi. org $/ 10.7326 / \mathrm{m} 15-2175$

Ree, M., Junge, M., \& Cunnington, D. (2017). Australasian Sleep Association position statement regarding the use of psychological/ behavioral treatments in the management of insomnia in adults. Sleep Medicine, 36, S43-S47. https://doi.org/10.1016/j.sleep. 2017.03.017

Seyffert, M., Lagisetty, P., Landgraf, J., Chopra, V., Pfeiffer, P. N., Conte, M. L., \& Rogers, M. A. M. (2016). Internet-delivered cognitive behavioral therapy to treat insomnia: A systematic review and meta-analysis. PLOS ONE, 11(2), e0149139. https://doi.org/ 10.1371/journal.pone.0149139

Shallcross, A. J., Visvanathan, P. D., Sperber, S. H., \& Duberstein, Z. T. (2019). Waking up to the problem of sleep: Can mindfulness help? A review of theory and evidence for the effects of mindfulness for sleep. Current Opinion in Psychology, 28, 37-41. https://doi.org/ 10.1016/j.copsyc.2018.10.005

Trauer, J. M., Qian, M. Y., Doyle, J. S., Rajaratnam, S. M. W., \& Cunnington, D. (2015). Cognitive behavioral therapy for chronic 
insomnia: A systematic review and meta-analysis. Annals of Internal Medicine, 163(3), 191-204. https://doi.org/10.7326/M14-2841

Walsh, J. (2004). Clinical and socioeconomic correlates of insomnia. The Journal of Clinical Psychiatry, 65(8), 13-19.

Watson, D., Clark, L., \& Tellegan, A. (1988). Development and validation of brief measures of positive and negative affect: The PANAS Scales. Journal of Personality and Social Psychology, 54(6), 1063. https://doi.org/10.1037/0022-3514.54.6.1063

Ye, Y.-Y., Chen, N.-K., Chen, J., Liu, J., Lin, L., Liu, Y.-Z., Lang, Y., Li, X.-J., Yang, X.-J., \& Jiang, X.-J. (2016). Internet-based cognitive-behavioural therapy for insomnia (ICBT-i): A meta-analysis of randomised controlled trials. British Medical Journal Open, 6(11), 1-10. https://doi.org/10.1136/bmjopen-2015-010707

Yook, K., Lee, S.-H., Ryu, M., Kim, K.-H., Choi, T. K., Suh, S. Y., Kim, Y.-W., Kim, B., Kim, M. Y., \& Kim, M.-J. (2008). Usefulness of mindfulness-based cognitive therapy for treating insomnia in patients with anxiety disorders: A pilot study. The Journal of Nervous and Mental Disease, 196(6), 501-503. https://doi.org/10. 1097/NMD.0b013e31817762ac
Zhang, B., \& Wing, Y.-K. (2006). Sex differences in insomnia: A metaanalysis. Sleep, 29(1), 85-93. https://doi.org/10.1093/sleep/29.1. 85

Zhang, J.-X., Liu, X.-H., Xie, X.-H., Zhao, D., Shan, M.-S., Zhang, X.-L., Kong, X.-M., \& Cui, H. (2015). Mindfulness-based stress reduction for chronic insomnia in adults older than 75 years: A randomized, controlled, single-blind clinical trial. Explore (NY), 11(3), 180-185. https://doi.org/10.1016/j.explore.2015.02.005

Publisher's Note Springer Nature remains neutral with regard to jurisdictional claims in published maps and institutional affiliations. 